\title{
Mythos
}

Rivista di Storia delle Religioni

$13 \mid 2019$

Varia

\section{Jesi e Wittgenstein: letture del mito tra simbolo e} senso

Jesi and Wittgenstein: Interpretations of Myth between Symbol and Meaning

\section{Marco Carmello}

\section{(2) OpenEdition \\ Journals}

Edizione digitale

URL: http://journals.openedition.org/mythos/783

ISSN: 2037-7746

\section{Editore}

Salvatore Sciascia Editore

Notizia bibliografica digitale

Marco Carmello, « Jesi e Wittgenstein: letture del mito tra simbolo e senso », Mythos [Online], 13।

2019, online dal 20 octobre 2019, consultato il 28 décembre 2019. URL : http://

journals.openedition.org/mythos/783

Questo documento è stato generato automaticamente il 28 dicembre 2019.

Mythos 


\title{
Jesi e Wittgenstein: letture del mito tra simbolo e senso
}

Jesi and Wittgenstein: Interpretations of Myth between Symbol and Meaning

\author{
Marco Carmello
}

\section{Introduzione}

1 In queste pagine si seguirà un filo apparentemente esile, ma non perciò irrilevante, della fitta trama intessuta da Furio Jesi nella sua lucida analisi dei fatti mitologici e letterari: quello della relazione col pensiero di Ludwig Wittgenstein. Limitata a due soli interventi, un articolo del 1972, poco più di una nota, ed un intervento più lungo del 1977, la questione sembra essere tanto marginale da non autorizzare neppure a parlare di una vera e propria relazione fra lo studioso torinese ed il filosofo austro-britannico, tanto più che Jesi non solo non è un filosofo del linguaggio, ma non ha mai mostrato interesse né per la filosofia analitica, né, al di fuori dei due luoghi qui considerati, per il pensiero complessivo di Wittgenstein.

2 Eppure, la natura dei problemi toccati nei due scritti dedicati a Wittgenstein e la loro connessione con alcuni dei nodi cruciali del pensiero di Jesi, primo fra tutti la definizione del mito come lingua entro i limiti del modello della macchina mitologica, impongono alla questione un'attenzione tanto più rigorosa quanto più la via lungo cui ci conduce il filo di queste riflessioni attorno al Viennese risulta essere chiarificatrice di alcuni aspetti forse a torto considerati troppo esoterici delle ricerche mitologiche di Jesi.

3 Le ragioni dell'interesse di Furio Jesi per il pensiero di Ludwig Wittgenstein non sono, dunque, né peregrine né facilmente definibili. Come si diceva, Jesi si occupa di Wittgenstein in due soli luoghi: l'articolo Prove di lettura del "limite" in Wittgenstein, apparso nel 1972 sulla rivista napoletana Logos, e quello apparso, cinque dopo, nel 1977, su Nuova corrente e poi compreso nella silloge Materiali mitologici dove si legge sotto il 
titolo Wittgenstein nei giardini di Kensington: le 'Bemerkungen über Frazers The Golden Bough' 1 .

4 L'attenzione verso il filosofo viennese coincide con lo sviluppo dell'ultima parte della produzione e del pensiero di Jesi, come paiono indicare le date dei due scritti di argomento wittgensteiniano che si pongono l'uno a ridosso di Mito, apparso nel 1973 per ISEDI, quando già è stato pubblicato Letteratura e mito (1968) ed è concluso Spartakus. Simbologia della rivolta ${ }^{2}$. Al periodo successivo, l'ultimo del percorso di Jesi, interrottosi nel 1980, appartiene invece il secondo degli scritti qui discussi. Dunque, l'interesse per Wittgenstein coincide con il momento in cui il concetto di "macchina mitologica" riceve la sua definitiva messa a punto iniziando così ad esercitare pienamente la sua funzione ermeneutica.

\section{Mito e mitologia: brevi considerazioni}

5 È lecito ricercare in un'intervista tarda, che va sotto il titolo di Quando Kerényi mi distrasse da Jung, le ragioni dell'interesse di Jesi per Wittgenstein:

Considerare la mitologia una interna e autonoma circolazione linguistica, peculiare di determinati materiali, significa da parte mia collocarmi al di fuori di correnti importanti e forse prevalenti della linguistica contemporanea, per le quali i materiali mitologici sono unicamente testi cui si attribuisce una qualifica "mitologici" solo perché, con un errore di metodo, si rimane ipnotizzati da una delle loro innumerevoli possibilità di lettura, e quella si privilegia come se disponesse di una sua intrinseca oggettività. Tale errore di metodo mi deriva da Kerényi [...] Non credo nell'esistenza del mito (uso la parola "credo" nel senso più forte, perché si tratterebbe propriamente di un atto di fede); sono invece convinto che per me, oggi, il modo migliore di collocarmi di fronte ai meccanismi e alle produzioni miei e degli altri, antichi o contemporanei, consista nel riconoscere in alcune di quelle produzioni un linguaggio non riducibile ad altri, assolutamente autonomo, "riposante in sé stesso" (Bachofen), dotate di altre caratteristiche definibili con approssimazioni estremamente vaghe se - com'è inevitabile per definirle - si ricorre ad altro linguaggio. (JESI 2013, 226-227) ${ }^{3}$.

La lunga, ma necessaria, citazione riportata dichiara in maniera diretta ed efficace la posizione di Jesi rispetto al mito, alla mitologia, alla lingua ed alle relazioni che si istituiscono fra questi tre fattori.

6 Mentre le prime righe della risposta che il mitologo dà al suo intervistatore stabiliscono un netto distacco fra l'opera di Jesi e l'analisi strutturalista, allora dominante nel quadro degli studi antropologici, delle mitologie, e dei sistemi linguistici - anche, se forse, sarebbe meglio dire "sistemi significanti" - che queste costituiscono, la seconda parte si incentra tutta sulle conseguenze che derivano dal fatto di considerare «la mitologia una interna e autonoma circolazione linguistica» anche, e soprattutto, in relazione alla definizione dei rapporti fra macchina mitica e linguaggio.

Si potrebbe quindi considerare questo testo dal punto di vista della mitologia, assumendo che "mitologia" sia il termine medio fra mythos ${ }^{4}$ e logos; da questo punto di vista appare chiaro l'implicito polemico della dichiarazione con cui Jesi si chiama fuori da «correnti importanti e forse prevalenti della linguistica contemporanea». L'autore torinese sta qui alludendo all'inevitabile, inevitabile almeno per Jesi, riduzionismo linguistico dell'analisi strutturalista del mito. 
8 La linea di pensiero di Jesi sembra poter essere così riassunta: se si assume di poter ridurre a testo un materiale mitico, leggendolo secondo un'ermeneutica che ricostituisce quel materiale stesso in quella forma, conferendogli così una strutturazione inesorabilmente linguistica, allora è inevitabile che la "miticità" stessa passi dall'essere un carattere inscindibile del materiale a diventare una caratterizzazione di un certo genere testuale. Fatto il passaggio dal materiale mitologico al testo mitologico, la lettura esclusivamente mitica del testo diviene un arbitrio interpretativo, poiché solo uno degli aspetti testuali, quello mitologico, viene preso in considerazione a discapito di tutti gli altri aspetti del testo, e, potremmo aggiungere noi esplicitando ancora meglio l'implicito presente nelle parole di Jesi, a discapito soprattutto di quegli aspetti sintattici che definiscono qualsiasi testo come rete di relazioni interne, indipendenti dai referenti, o meglio da ciò che l'operazione testuale estrania dal testo stesso definendolo come referente ${ }^{5}$.

In questo senso Jesi riduce ogni approccio strutturalista a una "linguistica" e nulla più. Il passaggio incriminato è proprio quello, primordiale, in forza di cui «i materiali mitologici» si trasformano «unicamente [in] testi»; è a questo primo passaggio linguistico che si deve quell'indistinzione fra mito e mitologia che marca la scienza del mito così come Jesi la descrive parlando di Lévi-Strauss (cfr.: JESI 2008: 115-126). Quest'indistinzione può essere interpretata come un esaurirsi del mito entro la mitologia, intesa come discorso (logos), più o meno pacificato, sul referente mito.

Se però il punto di partenza cui non si vuole rinunciare è l'assunzione dell'esistenza di una lingua mitologica intesa come lingua propria del mito, ossia di una mitologia "come interna ed autonoma circolazione linguistica», allora la trasformazione del materiale mitico in testo è esattamente quel che si vuole evitare. La testualizzazione del materiale mitico comporta infatti lo scadimento del mito stesso a testo, poiché quella testualizzazione non riconosce l'iscrizione dell'intraducibilità linguistica del mito entro i confini del linguaggio operata dalla nozione di «materiale mitologico» ${ }^{6}$.

11 Da qui le tre conseguenze del discorso jesiano cui l'autore allude nella seconda parte della sua risposta: la prima consiste nella postulazione del mito come primitivo linguistico irriducibile; la seconda consiste in una definibilità della lingua del "mito"; la terza consiste nella vaghezza di questa definibilità nella misura in cui sembra non essere possibile altro approccio alla definizione del mito che non sia quello che passa attraverso il linguaggio ${ }^{7}$.

12 Il fatto che il mito si presenti come un irriducibile linguistico, che sia, insomma, una lingua altra rispetto al linguaggio umano, ne impedisce quella riduzione a testo e racconto che invece segna in maniera peculiare la maggioranza degli approcci filologici ed etno-antropologici al mito stesso. Questa peculiarità linguistica irriducibile del mito, per parlare della quale Jesi ricorre al concetto bachofeniano di "simbolo riposante in sé stesso", quindi di simbolo auto-allusivo, solleva il problema della definibilità del mito stesso e quello della sua relazione col linguaggio.

Del resto, non avrebbe potuto essere altrimenti laddove si consideri che la definizione di un materiale mitico come irriducibile linguistico è anche l'unica che possa autorizzare a vedere la mitologia non più come riduzione linguistica del mito a racconto, dunque, in ultima analisi, a struttura, ma come circolazione linguistica interna ed autonoma. 

evitando di affrontare il problema di decidere se il pensiero di Jesi muova da posizioni solipsiste ${ }^{8}$, rimane pur sempre innegabile una postura metodologica fermamente internalista da parte dello studioso torinese nell'analisi dei fatti mitici.

15 È quindi questa postura metodologica e non funzionalista che determina il primo, e più importante, punto di contatto fra Jesi e Wittgenstein; non potrà, a questo punto, continuare a stupire che l'inizio di quella relazione parta dall'analisi di un aspetto del pensiero wittgensteiniano che parrebbe, ad una prima lettura, lontano dagli interessi di Jesi: il problema del limite così come questo viene a definirsi nel Tractatus 9 .

\section{Wittgenstein fra linguaggio e macchina mitologica} più di una ragione, scontata. Pur prendendo le mosse da un'interpretazione del cosiddetto "primo Wittgenstein", quello del Tractatus, l'articolo del 1972 definisce anche l'approccio al Wittgenstein autore delle Bemerkungen über Frazers The Golden Bough. Le note sul Ramo d'oro raccolgono una congerie di appunti compresi fra il 1930/31 ed il 1936/48, quindi, sebbene le Bemerkungen costituiscano un testo minore nel pensiero del filosofo austriaco, secondo l'esegesi tradizionale rappresentano un esempio del passaggio alla seconda fase dell'elaborazione filosofica di Wittgenstein, con cui si abbandonerebbe il solipsismo del Tractatus per porre le basi della filosofia del gioco linguistico e, quindi, della comunicazione.

17 Primo e secondo Wittgenstein, Wittgenstein del Tractaus e Wittgenstein delle Ricerche filosofiche, questa, come sappiamo, è sostanzialmente la base delle ermeneutiche accettate del pensiero del filosofo. Che si voglia porre il passaggio più in alto, collocandolo addirittura all'altezza dello stesso Tractatus, di cui la proposizione conclusiva, la famosissima proposizione 7: "Su ciò, di cui non si può parlare, si deve tacere", rendendo aporetico ogni tentativo di analisi interna del linguaggio, rappresenterebbe il punto di svolta verso una posizione pragmatica, oppure che si abbassi la cesura alla fase finale, composta dalle Ricerche logiche e da Sulla certezza, non cambia lo schema interpretativo: riconoscimento della crisi delle posizioni internaliste, congedo del solipsismo linguistico, creazione del concetto di gioco linguistico, definizione delle regole della comunicazione.

Ora, l'esegesi di Jesi, ponendosi a cavallo della cosiddetta svolta e postulando, sebbene implicitamente, un'assenza di frattura nella continuità di pensiero del Viennese, implica proprio la negazione di questo schema.

ne Jesi, come già si è detto, non sia un interprete del pensiero di Wittgenstein ${ }^{10}, \mathrm{i}$ punto di contatto che stabilisce fra la sua propria opera teorica e Wittgenstein è offerto da una zona ben precisa del pensiero del Viennese, una zona di rilevante interesse per il mitologo italiano, ma anche di fondamentale importanza per comprendere l'intento filosofico che anima il pensiero wittgensteiniano sul linguaggio, una zona che tende però a rimanere nascosta dietro l'apparato logico messo all'opera dalla ricerca sulle regole linguistiche intrapresa da Wittgenstein.

Il punto di contatto fra filosofo e mitologo è la symbolica, intesa come capacità dei sistemi semantici di mettere insieme senso e segno in unità significanti, i simboli appunto, a loro volta capaci di organizzarsi in strutture, in reti di rimandi coerenti 
organizzate sul doppio livello del linguaggio - i simboli parlano, dunque hanno una loro organizzazione sintattica - e del metalinguaggio: i simboli, oltre a "parlare" mediante l'attribuzione di senso al segno, secondo regole di conferimento ed attribuzione, e mediante la combinazione dei simboli così ottenuti secondo regole sintattiche, come avviene nel linguaggio, parlano anche di loro stessi in quanto simboli, comprendendo così il linguaggio medesimo fra i segni cui attribuire senso.

La relazione fra mito e linguaggio non è dunque, come proponevano Jakobson e LéviStrauss, la struttura, ma la capacità simbolica, la capacità cioè di elaborare su due livelli ciò che accade, definendo anzitutto una rete segnica e quindi attribuendo ai segni un senso simbolico.

Ma è proprio nell'analisi di questa capacità simbolica che si concentra tutta l'analogia fra il modello linguistico wittgensteiniano, così come è proposto nel Tractatus, ed il pensiero di Jesi: in gioco c'è quel modello del «girare in cerchio» (cfr. JESI 2008: 149-155) che caratterizza la macchina mitologica, fatta dal centro vuoto del mythos che irradia $\mathrm{i}$ suoi effetti sulla rete segnica della mitologia:

L'orizzonte sul quale si pone il modello macchina mitologica è lo spazio ove misuriamo questa perenne equidistanza da un centro non accessibile, rispetto al quale non si resta indifferenti, ma si è stimolati a stabilire il rapporto del "girare in cerchio". Questa nostra scelta è giustificata dalla necessità [...] di affrontare principalmente il problema della sostanza del mito [...] Mentre in codesto libro verificavamo di volta in volta [...] il modello girare in cerchio (e da ogni verifica traevamo elementi di conferma della coerenza e della necessità del modello che denominiamo macchina mitologica), sottolineavamo implicitamente la qualità ideologica della scelta di affermare o di negare la sostanza del mito. (JESI 2008, 149-150)

Attraverso queste parole, che si leggono all'inizio dell'Epilogo di Mito, possiamo scorgere il nesso occulto che la riflessione mitologica di Jesi ha con la filosofia linguistica di Wittgenstein. Anche l'analisi linguistica del Tractatus può essere definita come un "girare in cerchio", anzi, considerando quell'oltrepassamento della distinzione fra primo e secondo Wittgenstein, l'intero approccio, sia esso internalista, come nel Tractatus, oppure esternalista, come ormai richiesto dalle Bemerkungen, è un "girare in tondo".

Ma proprio qui si situa la rottura che separa nettamente Jesi da Wittgenstein e giustifica l'analogia: mentre la macchina mitologica è pensata espressamente per rifuggire dal problema della sostanza del mito, la filosofia di Wittgenstein presuppone una petizione di principio riguardo all'esistenza del linguaggio.

Wittgenstein esaurisce così la distanza fra circonferenza e centro, il suo "girare in tondo" si risolve nel ben noto gesto metafisico con cui il linguaggio parla di sé e su di sé, entrando nella sfera del "di più" linguistico di cui parla Melandri" ${ }^{11}$. Da qui la simbolicità inerente del pensiero del Viennese, per cui la logica è specchio del mondo, il suo chiudersi all'interno della macchina. Jesi coglie dunque uno dei tratti fondamentali della filosofia di Wittgenstein: l'impossibilità di considerare il linguaggio come modello ; la conseguenza è l'intrappolamento del Viennese entro il suo stesso gioco.

Nella prospettiva jesiana, dove è essenziale il rispetto delle misure, soprattutto il rispetto della forma cava della mitologia, intesa come modello che ruota intorno ad un centro che c'è ed è, simultaneamente, vuoto proprio perché ci si nega a fare supposizioni sulla sua sostanza, la macchina mitologica esiste come possibilità, prima ancora che come modello, di analisi, proprio perché: «La macchina mitologica, non 
appena cessa di essere considerata un puro modello funzionale e provvisorio, tende a diventare un centro fascinatorio e ad esigere prese di posizione, petizioni di principio circa il suo presunto contenuto» (JESI 2008, 154).

È esattamente quanto succede alla "macchina linguistica" di Wittgenstein, criticando la quale Jesi implicitamente stabilisce anche una relazione fra il mito e l'«altro linguaggio» che non può non essere di semplice allusione nella misura in cui il fatto di parlare del mito richiede uno spazio di vuoto indecidibile non solo rispetto alla verità del mito stesso, come già si è detto, ma anche rispetto alle regole di verità che costituiscono il linguaggio.

Solo in questo modo è possibile sfuggire alla trappola melandriana del "di più", evitando che il linguaggio parli per suo conto aprendo così la via anche alla generazione di una mitologia che simbolizza in forza delle sue regole. Il nesso fra metalinguaggio e mitologie secondarie, forse si potrebbe dire "metamitologie" era, del resto, ben chiaro a Jesi, come risulterà dalla sua lettura delle wittgensteiniane Bemerkungen, in cui invece il filosofo viennese non riesce a gestire le mitologie secondarie di Frazer, che pure individua, proprio perché incapace di vedere come queste facciano sistema con il metalinguaggio che inevitabilmente genera la sua macchina "mito-linguistica".

\section{Jesi lettore del Tractatus: I) cose (Dinge) e stati di fatto (Tatsachen)}

È quindi proprio la natura di macchina chiusa, altamente simbolica, del pensiero linguistico wittgensteiniano a richiamare l'attenzione di Jesi, per cui la macchina simbolica ha un senso solo nel momento in cui «[...] si offre all'io come matrice di pietre di confine, di limiti, di simboli che non dicono nulla e proprio perciò dicono in assoluto, abbandonano alla realtà mondo lo spazio infinito del loro dire, che è il dire» (JESI 2009: 187).

30 Ciò a cui dunque tende quella che noi abbiamo chiamato symbolica è il limite, la cui funzione è, rispetto alla symbolica stessa, quella di separare il linguaggio dal suo accadere, superando così, per via non dimostrativa, non logica, ma discorsiva ed attiva, il grande ed irresolubile problema del metalinguaggio. I simboli: «non dicono nulla e proprio perciò dicono in assoluto» (JESI 2009: 187); con quest'affermazione non si tratta né di proclamare l'inanità del simbolo, né di annullare l'attività significante in una sorta di misticismo insignificabile, ma di definire la funzione strategica assolta da quest'inanità. Il linguaggio dice, ma il suo dire, quel che potremmo chiamare l'accadere del linguaggio, non appartiene più al linguaggio ma al mondo, e rappresenta perciò uno "spazio infinito". Certo, noi possiamo reintrodurre parte di quello spazio nel linguaggio, possiamo cioè dare un segno - segnare - singoli accadimenti del linguaggio, singoli atti di dire, ma non il "dire" in assoluto, l'accadimento del linguaggio in quanto tale.

31 Il simbolo è dunque, anzitutto, frontiera interna al campo della lingua, fra linguaggio e "dire": con questa semplice affermazione Jesi in realtà cortocircuita l'intero Tractatus, mettendo in evidenza la relazione nascosta che esiste fra la prima e la settima proposizione: «Die Welt ist alles, was der Fall ist» ${ }^{12}$; «Wowon man nicht sprechen kann, darüber muß man schweigen» (WITTGENSTEIN 1992: 6 e 174) ${ }^{13}$. 

relazione fra la proposizione di apertura e quella di chiusura del Tractatus come segue: possiamo parlare del Mondo, ma solo perché, come specifica la proposizione seguente, ossia la 1.1.: «Die Welt ist die Gesamtheit der Tatsachen, nicht der Dinge» ${ }^{14}$ (WITTGENSTEIN 1992: 6), il mondo è un insieme di fatti, di realtà di cose che hanno già perso la loro natura di cosalità chiusa, ermetica. , iniziamo ad intravvedere che l'uso di Fall fa riferimento alla differenza SacheTatsache/Ding di cui parlavamo sopra. Fall, con il suo senso di casualità di ciò che accade $^{17}$ rappresenta la soglia oltre la quale, come ci dice la proposizione 2: «Was der Fall ist, die Tatsache, ist das Bestehehn von Sachverhalten» (WITTGENSTEIN 1992: 6) ${ }^{18}$. Dunque, passata la soglia, il fatto, ossia la cosa divenuta atto/azione gesto (Tat-sache), riceve un senso in quanto cosa organizzata, inserita e contenuta ${ }^{19}$ nella rete sintattica del linguaggio.

La Fall però, nel suo doppio senso di caso e di caduta, conserva un contatto con l'altro lato della soglia, il lato della Ding: la nozione di casualità di ciò che accade, di caduta della Ding, come cosa originariamente negata all'ordine logico del mondo, all'interno del Welt (mondo), e quindi la sua trasformazione in Tatsache ed in Sachverhalt (fatto/ ordine circostanziale), implica che sia proprio la Fall a non poter essere detta, quindi quello che non si può dire, e su cui dobbiamo tacere, è precisamente l'origine del linguaggio, e conseguentemente del mondo, che è fatto puramente linguistico nella misura in cui, come ci dice la seconda proposizione del Tractatus, ossia 1.1., esso è la totalità dei Tatsachen (dei fatti) e non delle Dinge (delle cose).

In quanto insieme di fatti il mondo è conoscibile e non pone né problemi di dicibilità, che non possano essere risolti da un uso corretto del linguaggio, né problemi di dottrina, poiché, come si dice nella proposizione 6.13: «Die Logik ist keine Lehere, sondern ein Spiegelbild der Welt. / Die Logik ist transzendental $\rangle^{20}$, dove per trascendentalità si intende il fatto che la logica, secondo quanto Wittegenstein scrive nel Tractatus, possa esaurire i Tatsachen (fatti) e, conseguentemente, anche il Mondo.

\section{Jesi lettore del Tractatus: II) la macchina chiusa}

Siamo così ritornati a Jesi: l'articolo del 1973; Jesi parte proprio da questa proposizione del Tractatus, ossia la 6.13, per dimostrare come nel pensiero di Wittgenstein si possa «riconoscere [...] lo sforzo di circoscrivere con appropriati strumenti linguistici una cosmologia mistica» (JESI 2009: 183). La chiave per giungere a quest'interpretazione, che, ricordiamolo, parte da una conseguenza inevitabile dell'approccio wittegensteiniano, ci viene data poco sotto: "immagine in uno specchio andrebbe inteso come un simbolo 'riposante in se stesso', come una tautologia, giacché nulla ci è detto e nulla è possibile dire circa l'eventualità che l'immagine sia immagine di qualcosa e che lo specchio 
simboleggi altro che se stesso» (JESI 2009: 183) ${ }^{21}$, dove vediamo all'opera anche un'espressione che Jesi prende da Bachofen.

L'analisi del pensiero di Wittgenstein parte dunque da un'indagine sulle conseguenze propriamente linguistiche dei presupposti primi della sua filosofia, che Jesi legge come forma di pensiero complessa, in cui si possono incrociare fra di loro ambiti di ricerca non omogenei: «la storia o scienza delle religioni, la filosofia, la matematica» (JESI 2009: 184). Il problema del limite è dunque anche interno al pensiero wittgensteiniano come problema della relazione fra i diversi ambiti da cui può essere affrontato o che lo compongono. Per Jesi, correttamente, non c'è un'integrazione - la disomogeneità degli ambiti resta inalterata ed irriducibile - c'è invece una linea di faglia comune costituita dal toccarsi delle frontiere di ambito, che disegnano così l'interpretazione del pensiero wittgensteiniano come non luogo. L'idea che la filosofia del Viennese si trovi a cavallo di ambiti disomogenei implica quella che il suo pensiero possa essere, o forse addirittura intenda costituirsi, come soglia fra ambiti differenti: quindi, a partire dall'interpretazione che ne dà Jesi, il pensiero di Wittgenstein assume l'aspetto di un passaggio, di una messa in relazione, ma non quello di un luogo.

Quello di Wittgenstein è però un sistema in sé chiuso, "mistico", dove per mistica si intende la completezza circolare e non dialettica della macchina. Jesi dimostra tale relazione mettendo in relazione fra di loro tre proposizioni del Tractatus secondo uno schema triadico che corrisponde a: dogma, commento del dogma e matrice del commento del dogma, secondo lo schema mitico, oppure, secondo uno schema logico più immediatamente vicino al testo del Tractatus, a mondo, realtà e sistema, dove sia realtà che sistema rappresentano un commento al mondo. Lo schema è questo:

\begin{tabular}{|l|l|l|l|}
\hline A & $\begin{array}{l}\text { 6.45 Die Anschauung der Welt sub specie aeterni ist ihre Anschauung als } \\
\text { - begrentzes - Ganzes. Das Gefühl der Welt als begrentzes Ganzes ist das } \\
\text { mystische. }{ }^{22} \text { (WITTGENSTEIN 1992, 172) }\end{array}$ & Dogma & Mondo \\
\hline B & $\begin{array}{l}\text { 4.463 Die Tautologie läßt der Wirklichkeit der ganzen - unendlichen - Commento } \\
\text { logischen Raum. }{ }^{23} \text { (WITTGENSTEIN 1992, 80) } \\
\text { al dogma }\end{array}$ & Realtà \\
\hline C & $\begin{array}{l}\text { Und wie wäre es auch möglich, daß ich er des Logik mit Forme zu tun } \\
\text { dieses System das logisch wichtige und nicht die einzelnen Symbole. / } \\
\text { hätte, die ich erfinden kann; sondern mit dem muß ich es zu tun haben, } \\
\text { was es mir möglich macht, sie zu erfinden. }{ }^{24} \text { (wITTGENSTEIN 1992, 130) }\end{array}$ & $\begin{array}{l}\text { Matrice del } \\
\text { commento }\end{array}$ & Sistema \\
\hline
\end{tabular}

Nel riordino proposizionale dato da Jesi all'ordine delle preposizioni del Tractatus colpisce il forte taglio subito dalla proposizione 4.463 , che funge da chiave di volta dello schema che abbiamo appena presentato. Le proposizioni del gruppo 4 originano dalla seguente proposizione: «Der Gedanke ist sinvolle Satz» ${ }^{25}$ (WITTGENSTEIN 1992: 40), ed analizzano la relazione fra proposizione, mondo e valori di verità, come segue:

Die Wahreitsbedingungen bestimmen den Spielraum, der den Tatsachen durch den Satz gelassen wird (Der Satz, das Bild, das Modell, sind in negativen Sinne wie ein fester Körper, der die Bewegungsfreiheit des andern beschränkt; im positiv Sinne, wie der von fester Substanz begrenzte Raum, worin ein Körper Platz hat). Die Tautologie läßt der Wirklicheit den ganzen - unendlichen - logischen Raum; die Kotradiktion erfült den ganzen logischen Raum und läßt der Wirklichkeit keinnen 
Punkt. Keinen von beiden kann deher die Wirklichkeit irgendwie bestimmen.

(WITTGENSTEIN 1992, 80) ${ }^{26}$

ha a che fare con la sua presentazione del "misticismo wittgensteiniano", ma è in realtà irrilevante rispetto allo schema di ragionamento che egli propone. Sia secondo l'ordine del "mito" sia secondo quello "matematico" dell'assiomatica, il commento, cioè la proposizione $\mathrm{B}$, è ridondante poiché già di per sé stesso il dogma dovrebbe essere autoevidente, cioè a dire tautologico per Jesi - tautologico oppure contraddittorio, seguendo più da vicino Wittgenstein (tanto la tautologia come la contraddizione sono auto-evidenti) - quindi, dall'autoevidenza di B, segue che la regola linguistica da cui si ricavano i simboli, quei simboli che non possono essere inventati, come ci dice $C$, faccia aggio sul dogma, cioè sulla proposizione $\mathrm{A}$, chiudendo così lo schema in un circolo argomentativo in cui inizio e fine coincidono. dell'enigma del mondo attraverso lo specchio linguistico, anche se, ed è qui la ragione più profonda del fascino che il pensiero di Wittgenstein esercita su Jesi, il filosofo viennese giunge estremamente vicino a compiere il passo finale di un'emancipazione del pensiero dalla teoria.

Non è dunque scorretto indicare, come primo e più profondo gesto teorico, proprio quella spezzatura fra linguaggio e metalinguaggio che, segnando il linguaggio stesso per attribuirgli un senso, ne fa un simbolo esposto ad uno dei più complessi atti "teorici" di visione, quello che si risolve in una visione della visione stessa con cui l'immagine di uno specchio si riflette, a perdita d'occhio, in quella di un altro, uguale e contrapposto, specchio.

\section{Intorno alle Bemerkungen über Frazers "The Golden Bough"}

Il passaggio ad una symbolica, quindi, pare suggerire Jesi, l'intento di uscita dal metalinguaggio che Wittgenstein sarebbe stato sul punto di compiere, senza tuttavia attuarlo effettivamente, risulta evidentemente bloccato da un'impossibilità da parte di Wittgenstein a compiere fino in fondo il passo ultimo verso il dovere di "dissodare l'intero linguaggio" per scoprire così "l'intera mitologia" che in esso si è depositata, come scrive il filosofo viennese e nelle sue Osservazioni su Frazer ${ }^{27}$.

Dunque, quando cinque o sei anni dopo l'articolo del 1973, Jesi ritorna, apparentemente da tutt'altro punto di vista, a considerare il pensiero di Ludwig Wittgenstein, in un articolo originariamente apparso su Nuova Corrente, poi significativamente ${ }^{28}$ incluso in quel vero e proprio laboratorio che è costituito dai Materiali mitologici intitolato Wittgenstein nei giardini di Kensington: le Bemerkungen über Frazers "The Golden Bough", la prospettiva è già segnata.

Di questo secondo articolo prenderemo in rapida considerazione solo due affermazioni, una posta al principio, in cui Jesi dichiara il suo intento scrivendo: «La cosa più interessante ci sembra [...] studiare le Bemerkungen [...] come documento dell'incomprensione di Wittgenstein nei confronti di Frazer, o eventualmente della sua comprensione più o meno implicita, ma anche del suo rifiuto assolutamente esplicito» (JESI 1979: 158); l'altra alla fine, dove Jesi ci dice che: 
Le due sentenze di Wittgenstein: Wir müssen die ganze Sprachen durchpflugen; In unsere Sprache iste eine ganze Mythologie niedergelegt ${ }^{29}$ sono anzitutto indicazioni metodologiche che mirano a difendere dall'attività mitogenetica degli esplicatori della magia ed a conservare la sua autonoma Tiefe (profondità). La magia diventa profondamente vera e terribile quando si innesta, oggi, su di essa un processo mitogenetico per opera di coloro che vogliono dire la verità sulla magia. L'orrore nei confronti di questa mitogenesi è l'impulso a dissodare l'intera Sprache per individuarvi i semi mitologici ancora vitali che vi si trovano 'niedergelegt' (lasciati/ posati) e difendersi da chi si proponesse di farli germinare in inquietanti spazi quotidiani di esoterismo da serra (JESI 1979, 173)

Come è noto, Jesi riporta l'operazione antropologica di sir James a quell'Inghilterra tardo-vittoriana ed Edoardiana in cui nasce la passione per l'esoterismo, per le raccolte di errori/orrori, con cui, ormai già dentro la pienezza della modernità, l'uomo tardo ottocentesco e primo novecentesco arredava un ambiente culturale di sottile sovversione, il cui intento era quello di creare, attraverso un'estetizzazione del mito, il terreno utile alla giustificazione ideologica di un certo ordine di cose.

In questo senso, come sostiene Wittgenstein, la spiegazione evolutiva data da Frazer risulta più terribile del mito stesso, proprio perché, come ormai possiamo dire anche noi sulla scorta di Jesi, spesso si tratta di una spiegazione più efferatamente ideologica del mito, di una mitologia seconda, fondata su un'altra mitologia attraverso un processo pseudoscientifico di invalidazione delle credenze primitive e di rilancio (pseudo-)scientifico di strutture mitologiche.

Se dunque è vero che Wittgenstein coglie il pericolo di approcci alla Frazer, al di là delle critiche superficiali, è però anche vero che il filosofo è ancora trattenuto al di qua delle conseguenze cui questa coscienza dovrebbe portarlo proprio dalla mistica della sua symbolica semantica e formale ma non oggettuale. Si tratta di quel gesto descritto da Rush Rhees nella sua introduzione, quando ci dice che la sezione dattiloscritta su Frazer, quella comprendente gli appunti del 1930/31 iniziava, nella versione manoscritta, con tre affermazioni poi cassate da Wittgenstein, che appose in margine alle tre frasi una S, abbreviazione di "schlecht" (catttivo) ed evitò di farle copiare (WITTGENSTEIN 1967: 233). L'inizio cassato suona così:

Ich glaube jetz, daß es richtig wäre, mein Buch mit Bemerkungen über die Metaphysik als eine Art von Magie zu beginnen. / Worin Ich aber weder die Magie das Wort reden noch mit über sie lustig machen darf. / Von der Magie müßte die Tiefe behalten werden. - / Ja das Ausschalten der Magie hat hier den Charakter der Magie selbst. /Denn, wenn ich damals anfing von der 'Welt' zur eden (und nicht von diesem Baum oder Tisch), was wollte ich anderes als etwas Höeres in meine Wrote bannen. (WITTGENSTEIN 1967, 233-234) 30 $^{30}$

Rhees scrive che possiamo capire il perché dell'esclusione ${ }^{31}$, ed effettivamente ha ragione: se Wittgenstein accettasse veramente di parlare di mito dovrebbe lasciare da parte i fatti, i Tatsachen per parlare di cose, di Dingen, superando così quell'interdetto a non dire ciò di cui non c'è niente da dire che, come Jesi ci insegna, Wittgenstein effettivamente non ha mai superato. La scelta di una strategia esclusivamente formale e semantica nel processo di attribuzione di senso al segno condanna il filosofo viennese ad una dimensione mistica insuperabile, sulla quale si fonda l'unità profonda di tutta la filosofia wittegensteiniana, dal Tractatus alle Ricerche, senza alcuna differenza fra un primo ed un secondo tempo.

51 Ma la risoluzione del linguaggio in una symbolica capace di spiegarne il portato mitologico era per Wittgenstein impossibile, proprio perché quella costituzione delle 
cose in stati di fatto all'interno del linguaggio implica un cedere al fascino della macchina simbolica della lingua e del mito, affermando, implicitamente ma non per questo meno fortemente, l'esistere delle cose. La via di una reale critica linguistica resta così, almeno secondo Jesi, serrata al grande analista del linguaggio stesso. Sarà invece il mitologo a prenderne il posto in forza della vuotezza provvisoria della macchina mitologica, capace di bloccare il fascino simbolico di miti e parole.

\section{BIBLIOGRAFIA}

BOUVERESSE (1997): J. Bouveresse, Filososfia, mitologia e pseudoscienza, Torino 1997.

CAVALLETTI (2001): A. Cavalletti, «La maniera compositive di Furio Jesi», in Materiali mitologici. Mito e antropologia nella cultura mitteleuropea, Torino 2002, 357-376.

CLACK (1999): B. R. Clack, Wittgenstein, Frazer and Religion, London - New York 1999.

Coveos (1990): C. M. Coveos, «Wittgenstein on Frazer's The Golden Bough», Philosophy 65 (1990), 518-521.

DAVIES (1983): P. Davies, «Remarks on Wittgenstein's 'Remarks on Frazer's The Golden Bough'», King's Theological review 6, 1 (1983), 518-521.

DUMMETT 1993: R. Dummett, Origins of Analytical Philosophy, London 1993.

HADOT 2004: P. Hadot, Wittgenstein et les limites du language, Paris 2004.

HINTIKKA (1958): J. Hintikka, «On Wittegenstein's ‘Solipsism'», Mind 67 (1958), 88-91.

JESI 2001: F. Jesi, Materiali mitologici. Mito e antropologia nella cultura mitteleuropea, nuova edizione a cura di Andrea Cavalletti, Torino 2001 (ed. or. Torino 1979).

JESI 2008: F. Jesi, Mito, nuova edizione con una Nota di G. Schiavoni, Torino 2008.

JESI 2009: F. Jesi, «Prove di lettura del 'limite' in Wittgenstein», Nuova Corrente 56 (2009), 183-188.

JESI 2013: F. Jesi, «Quando Kerényi mi distrasse da Jung», in F. Jesi, Il tempo della festa, Roma 2013, 224-231.

KENNY 1973: A. Kenny, Wittgenstein, London 1973.

MANERA 2012: E. Manera, Furio Jesi. Mito, violenza, memoria, Roma 2012.

MELANDRI 2007: E. Melandri, Contro il simbolico. Dieci lezioni di filosofia, Macerata 2007.

PERISINOTTO 2008: L. Perisinotto, Wittgenstein. Una guida, Milano 2008.

SACHS (1988): «On Wittegenstein's Remarks on Frazer's Golden Bough», Philosophical Investigations 15, 2 (1988), 147-150.

SBISÀ (1984): M. Sbisà, «Frazer e Wittgenstein. Pathos delle spiegazioni causali e mistica della descrizione fisioniomica», La ricerca folklorica 10 (1984), 33-40.

SCHUlTE (2006): J. Schulte, Coro e legge. Wittgenstein e il suo contesto, Lecce 2006. 
SCHULTE (2019): J. Schulte, Wittgenstein. Un'introduzione, Macerata 2019.

SLUGA 2004: H.G. Sluga (edited by), The Cambridge Companion to Wittgenstein, Cambridge 2004.

STERN (1996): D.G. Stern, Wittgenstein on Mind and Language, Oxford 1996.

WITTGENSTEIN 1967: L. Wittgenstein, «Bemerkungen über Frazers The Golden Bough», Synthese 17

(1967), 233-253.

WITTGENSTEIN 1975: L. Wittgenstein, Note sul "Ramo d'oro" di Frazer, con un saggio di J. Bouveresse, trad. it. di S. De Waal, Milano 1975.

WITTGENSTEIN 1992: L. Wittgenstein, Tractatus logico-philosophicus, a cura di A.G. Conte, Torino 1992.

ZALABARDO (2015): J. L. Zalabardo, Representation and Reality in Wittgenstein's Tractatus, Oxford 2015.

\section{NOTE}

1. Prove di lettura del "limite" in Wittgenstein si legge originariamente in Logos 1973, n. 2, alle pp. 274-278 e viene successivamente riproposto nel 2009 su Nuova corrente 56, pp. 183-188 (è quest'ultima la versione da cui qui si cita); il breve scritto, per dimensioni, ma non per densità ed importanza, poco più di una nota, non viene poi riproposto in altra sede. Wittgenstein nei giardini di Kensington è compreso alle pagine 158-173 dei Materiali mitologici, pubblicati da Einaudi nel 1979, ed originariamente era apparso, nel 1977, in Nuova corrente, 72-73, alle pp. 168-183.

2. L'opera è già pronta nel 1969, ma avrà una storia editoriale particolarmente sfortunata, tanto che il testo sarà pubblicato solo nel 2000 (JESI 2013, 7-9); per quanto riguarda invece i Materiali mitologici rimando all'appendice curata da Andrea Cavalletti alla riedizione einaudiana dell'opera (JESI 2001, 335-356). Rimando inoltre all'importante postfazione di Cavalletti allo stesso volume, La maniera compositiva di Furio Jesi (CAVALLETTI 2001, 357-376) per l'inquadramento dell'articolo entro la stratificata silloge dei materiali; la discussione delle molteplici relazioni che il testo su Wittegenstein lettore di Frazer intrattiene con la raccolta dei Materiali esulano infatti dal limite puntuale di quest'articolo. Nella non estesissima bibliografia critica su Jesi è da segnalare, almeno, MANERA 2012, in particolare il primo paragrafo del quinto capitolo, dedicato a Rilke, Benjamin e Wittgenstein (MANERA 2012, 125-131), che permette un inquadramento generale delle riflessioni su Wittgenstein, argomento esclusivamente monografico di questo scritto, entro il più ampio quadro del pensiero jesiano.

3. Il testo dell'intervista apparve, per la prima volta, in Alias 30, 2007, p. 21. Secondo quanto scrive Cavalletti nella nota al testo (JESI 2013, 223), si tratta di uno degli ultimi testi dell'autore; rimasta senza titolo, l'intervista ricevette quello attuale da parte della redazione di Alias (JESI 2013, 223). Vengono conservati corsivi e virgolette dell'originale.

4. L'uso del termine greco risponde alla differenza che Jesi stesso, in Mito (JESI 2008, 13-36), stabilisce fra: mythos come originario valore religioso, e "mito" come portato culturale susseguente all'età greca.

5. La posizione che si ricava dall'intervista rappresenta una radicalizzazione di quanto Jesi aveva scritto in Mito (JESI 2008, 115-126) agli inizi degli anni '70; in realtà, però, già da quella data l'indagine di Jesi andava contro la "linguisticizzazione" del mito implicita nell'approccio strutturalista.

6. Alla nozione, per nulla scontata o semplice, di materiale mitologico andrebbe dedicato un saggio a parte. Secondo l'interpretazione qui assunta, il materiale mitologico è quella forma che il mito prende quando viene assunto come oggetto di un discorso; la peculiarità di questa nozione consiste, però, nel fatto di assicurare l'inesauribilità linguistica del mito. Il materiale mitologico 
segna dunque i limiti del linguaggio nella misura in cui si presenta come una costruzione segnica autonoma, un simbolo riposante in sé stesso, direbbe Jesi sulla scorta di Bachofen, che comporta una quidditas irriducibile alla struttura linguistica. Il materiale mitologico è così il segno dell'intraducibilità linguistica del mito stesso, almeno secondo la nostra lettura. Poiché la differenza, pur tanto importante per la retta comprensione del pensiero di Jesi, fra mito e materiale mitologico non gioca, nel contesto specifico del nostro discorso, un ruolo rilevante, e l'effetto della riduzione del materiale mitologico a testo è la testualizzazione del mito stesso, nel prosieguo parleremo di riduzione del mito a testo ; su queste questioni rimando sempre a MANERA 2012.

7. In realtà Jesi parla genericamente di «altro linguaggio», ma è facile intendere, rispetto al contesto entro cui si sviluppa il suo discorso, che l'altro linguaggio, rispetto a quello del mito, sia la lingua naturale.

8. Proprio analizzando la posizione di Jesi verso il pensiero di Wittgenstein vedremo che non è così, il discorso jesiano assume infatti una dimensione dialettica che nega nettamente posizioni di tipo solipsista, anche se, ed è forse questo uno degli elementi di maggior novità di Jesi, lo fa a partire da un'analisi interna e non funzionale del fatto mitico.

9. Dall'ampia bibliografia sul Tractatus segnalo il recente ZALABARDO 2015, tengo sullo sfondo le considerazioni raccolte in HINTIKKA 1958. Sul problema del limite linguistico si vedano: STERN 1996, e, anche se da tutt'altro punto di vista, HADOT 2004. Per una presentazione generale si vedano invece il classico KENNY 1973, SLUGA 2004 e SCHULTE 2019, può essere utile indicare anche il presentativo, ma interessante, PERISSINOTTO 2008.

10. Evidentemente il Torinese non è interessato ad una lettura approfondita del percorso filosofico del Viennese, anzi, l'impressione che si ricava leggendo quanto Jesi scrive su Wittgenstein è quella della lettura approfondita ma puntuale, intesa a chiarire aspetti specifici dell'oggetto d'analisi, piuttosto che ad inserire quest'ultimo all'interno di un quadro interpretativo generale, che tenga conto dell'evoluzione e della dialettica interna all'oggetto stesso.

11. Cfr. MELANDRI 2007, 39-66.

12. WitTGENSTEIn 1992, 7: «Il mondo è tutto ciò che accade». Per le traduzioni in italiano delle citazioni tratte dal Tractatus si ricorre alla traduzione di A. G. Conte. Le citazioni, come le traduzioni, riproducono fedelmente l'usus scribendi dell'originale. L'uso del segno / indica gli a capo presenti nella fonte.

13. WITTGENSTEIN 1992, 175: «Su ciò, di cui non si può parlare, si deve tacere».

14. WITTGENSTEIN 1992, 7: «Il mondo è la totalità dei fatti, non delle cose».

15. Ma è proprio nella definizione del Tatsache generato dal fallen della Ding entro il linguaggio che deve vedersi uno dei momenti di chiusura della macchina wittgensteiniana, poiché inevitabilmente l'organizzarsi della cosa in stato di fatto entro il linguaggio implica che si assuma una posizione sulla sostanza, come direbbe Jesi, della cosa stessa.

16. In luogo di geschehen o vorkommen (passieren non apparteneva al registro di tedesco usato da Wittgenstein).

17. Si consideri la proposizione 1.21: «Eines kan der Fall sein oder nicht der Fall sein und alles übrige gleich bleiben» (Qualcosa può accadere o non accadere, e tutto ciò che resta rimanere uguale). (WITTGENSTEIN 1992, 6-7)

18. «Ciò che accade, il fatto, è il sussister [l'esistere, l'essere] di stati di cose [circostanze di fatto, fatti]».

19. Verhalten vuol dire "contenere", "reprimere" e die Verhalten è la condotta nel senso di contegno.

20. «La logica non è una dottrina, ma un'immagine speculare del mondo. La logica è trascendentale.» 


\section{Corsivi nell'originale.}

22. WITTGENSTEIN 1992, 173: «Intuire il mondo sub specie aeterni è intuire il mondo quale tutto limitato -. Sentire il mondo quale tutto limitato è il mistico».

23. WITTGENSTEIN 1992, 81: «La tautologia lascia alla realtà tutto - infinito - lo spazio logico».

24. WITTGENSTEIN 1992, 131: «Ma dove si possono formare simboli secondo un sistema, ivi questo sistema è ciò che è logicamente importante, e non i simboli. E come sarebbe possibile che nella logica io avessi a che fare con forme che posso inventare? Io devo invece aver a che fare con ciò che mi rende possibile inventarle».

25. WITTGENSTEIN 1992, 41: «Il pensiero è la proposizione munita di senso».

26. WITTGENSTEIN 1992, 81: «Le condizioni di verità determinano lo spazio, che attraverso la Proposizione viene lasciato ai fatti (La proposizione, l'immagine, il modello, sono in senso negativo come un corpo solido, che limita la libertà di movimento di un altro corpo; in senso positivo, come lo spazio limitato da una sostanza solida in cui un corpo ha posto). La tautologia lascia alla realtà l'intero - illimitato - spazio logico; la contraddizione occupa l'intero spazio logico e non lascia alla realtà alcun punto. Nessuna delle due, dunque, può in qualche modo determinare la realtà».

27. Oltre al saggio di Boveresse la cui traduzione è pubblicata in appendice all'edizione italiana delle note di Wittgenstein (WITTGENSTEIN 1975, 63-90), segnalo BOUVERESSE 1997 CLACK 1999, COVEOS 1990, DAVIES 1983, SACHS 1988, SBISÀ 1984, SCHULTE 2006; l'insieme di queste opere dà un quadro generale abbastanza soddisfacente dei temi inerenti l'antropologia, la psicologia, le credenze mitiche e religiose, l'etnologia che formano l'altra faccia della riflessione di Wittgenstein. Anche in questo caso l'intento escussivo dissuade da un inquadramento delle note frazeriane entro il pensiero del filosofo. Qui ci interessano esclusivamente gli aspetti inerenti alla lettura di Jesi.

28. Dico significativamente almeno per due ragioni; la prima, esterna, è che, in netto anticipo su Dummett (DUMmetт 1993), Jesi riconduce proprio all'incubatrice il pensiero di Wittgenstein, indicando così una strada di valida comprensione di alcuni temi propri della filosofia analitica, la seconda, interna, è che la collocazione di Wittgenstein in un contesto mitteleuropeo rappresenta una sottile, ma a nostro giudizio chiara, presa di posizione sull'intera elaborazione filosofica di area germanofona.

29. «Dobbiamo dissodare l'intero linguaggio» e «Nel nostro linguaggio si è posata un'intera mitologia» (WITTGENSTEIN 1975, 27 e 31); per le Bemerkungen si segue la traduzione italiana di S. De Waal.

30. WITTGENSTEIN 1975, 12: «Io credo che ora sarebbe giusto cominciare il mio libro con alcune considerazioni sulla metafisica considerata come tipo di magia. / Nel far questo non potrei però né prendere le parti, né irriderla. / Della magia bisognerebbe mantenere la profondità / Sì, l'esclusione della magia ha qui il carattere della magia. / Perché allora mi sono messo a parlare del 'mondo' (e non di questo albero o di questo tavolo), che altro potevo volere se non catturare qualcosa di più alto nelle mie parole?».

31. WITTGENSTEIN 1967, 233: «The typed section on Frazer begins with three remarks which are not connected with them in the manuscript. He had begun there with remarks which later marked $\mathrm{S}$ (= 'schlecht') and did not have typed. I think we can see why». 


\title{
RIASSUNTI
}

Il presente articolo si occupa del ruolo che l'interpretazione del pensiero di Wittgenstein ha nella definizione del modello di "macchina mitologica" da parte di Furio Jesi. Ci si concentra soprattutto sui due interventi da Jesi dedicati al filosofo viennese nel 1972 e nel 1977.

This article discusses the importance of the Wittgenstein's thought for the definition of the model of the "mythological machine" in Jesi's work. We focus particularly on the two articles written by Jesi on Wittgenstein in 1972 and 1977.

\section{INDICE}

Keywords : language, mythological machine, symbol, Jesi Furio, Wittgenstein

Parole chiave : linguaggio, macchina mitologica, simbolo, Jesi Furio, Wittgenstein

\author{
AUTORE \\ MARCO CARMELLO \\ Universidad Complutense de Madrid \\ Departamento de Estudios Románicos, Franceses, Italianos y Traducción, \\ Facultad de Filología, Edificio D, desp. 359 - planta segunda, Ciudad Universitaria s/n, \\ 28040 Madrid \\ macarmel(at)filol.ucm.es
}

